\title{
Dynamic Surface Enrichment in Drying Thin-Film Binary Polymer Solutions
}

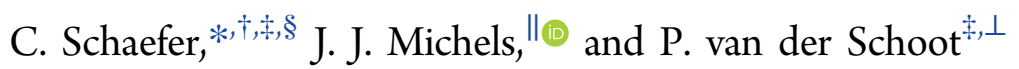

${ }^{\dagger}$ Dutch Polymer Institute, P.O. Box 902, 5600 AX Eindhoven, The Netherlands

*Theory of Polymers and Soft Matter, Eindhoven University of Technology, P.O. Box 513, 5600 MB Eindhoven, The Netherlands

${ }^{\S}$ Simbeyond B.V., Groene Loper 19, 5612 AE Eindhoven, The Netherlands

"Max Planck Institut für Polymerforschung, Ackermannweg 10, 55128 Mainz, Germany

${ }^{\perp}$ Institute for Theoretical Physics, Utrecht University, Princetonplein 5, 3584 CC Utrecht, The Netherlands

\section{Supporting Information}

ABSTRACT: Solution-cast, thin-film polymer composites find a wide range of applications, such as in the photoactive layer of organic solar cells. The performance of this layer crucially relies on its phase-separated morphology. Efficient charge-carrier extraction requires each of the components to preferentially wet one of the two electrodes. It is often presumed that the experimentally observed surface enrichment required for this is caused by specific interactions of the active ingredients with each surface. By applying a generalized diffusion model, we find the dynamics to also play an important role in determining which component accumulates at which surface. We show that for sufficiently fast evaporation the component with the smallest cooperative diffusivity accumulates at the free interface. Counterintuitively, depending on the interactions between the various components, this may be the smaller solute. Our comprehensive numerical and analytical study provides a tool to predict and control phase-separated morphologies in thin-film polymer composites.

\section{INTRODUCTION}

Solution-cast thin-film polymer composites find a wide range of applications, such as in the photoactive layer of organic solar cells. ${ }^{1-7}$ The performance of this layer crucially relies on its phase-separated morphology, in which the size of the domains of the electron donating and accepting components is ideally of the order of the exciton-diffusion length. On top of that efficient charge-carrier extraction requires the two components be in direct contact with the opposite electrodes.

The composite films are manufactured by casting a solution onto a solid substrate, after which the solvent is removed by rapid evaporation. During evaporation a mesoscopic structure emerges in the solution, characterized by local enrichment or depletion of either component. The detailed morphology of the final, dry film depends sensitively not only on the properties of the various components and the substrate but also on the rate at which the solvent is extracted. ${ }^{8}$ Prior to kinetic arrest, caused by lack of solvent, the structure that develops is caused on the one hand by demixing processes and on the other by stratification. Demixing may involve the precipitation of solids 9 or fluid-fluid phase separation..$^{5-7,10-12}$ Depending on the temperature and composition, liquid-liquid demixing may occur via spinodal decomposition or nucleation and growth, while the precipitation of solid components is by nucleation and growth. Stratification may be induced by interaction of one

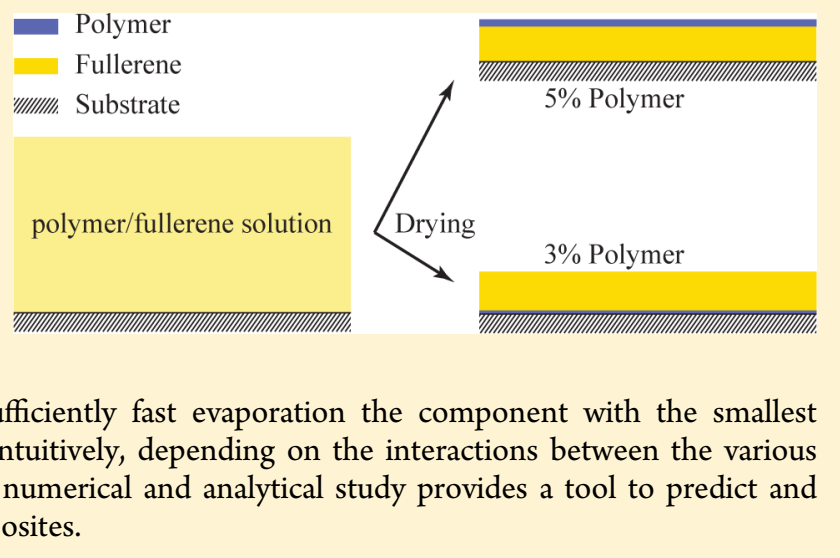

or more of the components with the substrate or by evaporation, leading to mass accumulation near the free surface.

The interplay between demixing and stratification may lead to lateral phase separation close to the free surface ${ }^{13}$ or may first lead to vertical demixing into phase-separated lammelae that eventually break up due to Marangoni instabilities. ${ }^{14-16}$ Current three-dimensional phase-field models have included evaporation, multicomponent diffusion, hydrodynamics, ${ }^{16}$ evaporation, ${ }^{11,17}$ thermal fluctuations, ${ }^{7}$ and surface roughening $^{18,19}$ and are capable of qualitatively explaining experimentally encountered dry-layer morphologies. Still, the parameter space is enormous, and quantitative comparison between model and theory remains rare, if not absent.

In order to proceed toward a more quantitative understanding of morphology formation, it is crucial to to understand the physical principles that underly the formation of vertically demixed lamellae. Some important progress has been provided by Wodo and Ganapathysubramanian, ${ }^{12}$ who have identified four modes of vertical phase separation. These include mechanisms where spinodal decomposition (i) is uniform, (ii) is directed by the substrate, (iii) is directed by the free surface, and (iv) occurs through a competition of the latter two.

Received: June 9, 2017

Published: July 18, 2017 
What is lacking in the discussions of theoretical studies is which of the solute components enriches the free surface and why.

Selective surface enrichment has been argued to originate from preferential wetting of components at the substrate surface and at the free surface. ${ }^{12,20-24}$ While not implausible, this begs the question to what extent dynamical processes are involved in particular in determining the composition at the free interface. Indeed, in the case of binary colloidal mixtures dispersed in a an evaporating solvent, the moving interface mops up the dispersed particles if these cannot diffuse out of the way swiftly enough. ${ }^{25}$ In solute mixtures this effect could at some point lead to phase separation, initiated by the moving interface. $^{12}$

In this work, we apply a diffusion-evaporation theory to a binary solution, modeled on an actual polymer/fullerene/ solvent mixture used for fabricating organic solar cells. In agreement with recent findings on evaporating colloidal dispersions, ${ }^{25,26}$ we find that for sufficiently fast evaporation the component with the smallest cooperative diffusivity accumulates at the free interface. Which component is slowest is dictated by both the concentrations and the interactions, mediated by the (cross) diffusivities. ${ }^{26}$ This implies that the smaller (fullerene) rather than the larger (polymer) solute may be slowest and may accumulate at the surface. In contrast to the colloidal mixtures considered in refs 25 and 26, our polymer mixture phase separates when the surface concentration reaches the spinodal concentration. We show that the values of the cooperative diffusivities at this concentration determine what component enriches the surface.

Our comprehensive numerical and analytical study predicts under what conditions what component enriches the free surface, which implies that phase-field models can be experimentally verified by simply studying the surface enrichment rather than the full three-dimensional dry-layer morphology. The remainder of this article is arranged as follows. We first present a generalized diffusion model to describe vertical phase separation and evaporation. Next, we evaluate the model numerically and show surface enrichment to occur despite the absence of surface interactions. We then explain this phenomenon by deriving an approximate theory, which predicts for each solute the conditions for which it enriches the liquid-vapor interface.

\section{GENERALIZED DIFFUSION-EVAPORATION MODEL}

We set up our theory by considering a thin film, consisting of a polymer $\mathrm{A}$ and a fullerene $\mathrm{B}$ in a solvent $\mathrm{S}$. We specify our model compounds below. The thickness $h(t)$ of the thin film decreases with time, $t$, due to solvent evaporation. We take as elementary length scale the size of a solvent molecule, and the thickness $h$ we express in units of this length scale and hence is dimensionless. In the remainder of this article, all units of length and time are expressed in these units. Plausibly, the speed at which the thickness of the fluid film decreases is proportional to the volume fraction of solvent at its surface. $^{11,17,27}$ From this evaporation rate at time zero and from the initial (dimensionless) film thickness $h_{0} \equiv h(0)$, we obtain an approximate time at which the film reaches dryness. We make time dimensionless by dividing it by this evaporation time; i.e., the film is dry at time $t \approx 1$.

We presume the solution to be incompressible and consequently the sum of the volume fractions of all the components, $\phi_{\mathrm{A}}, \phi_{\mathrm{B}}$, and $\phi_{\mathrm{S}}$, equals unity: $\sum_{m=\mathrm{A}, \mathrm{B}, \mathrm{S}} \phi_{m}=1$. For simplicity, the volume fractions depend exclusively on the distance $z$ from the substrate, which runs from 0 to $h(t)$, and on time $t$. Because of our incompressibility assumption, we do not need to set up three dynamical equations but only two.

We choose to focus on those for $\phi_{\mathrm{A}}$ and $\phi_{\mathrm{B}}$ of the solutes $\mathrm{A}$ and $\mathrm{B}$. Let the mass transport of both components be entirely diffusive and isothermal. If that is the case, then the dynamics of mass transport is governed by the following quasi-one-

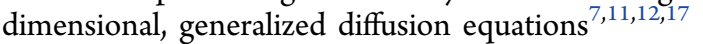

$$
\partial_{t}\left(\begin{array}{l}
\phi_{\mathrm{A}} \\
\phi_{\mathrm{B}}
\end{array}\right)=\partial_{z}\left[\left(\begin{array}{cc}
M_{\mathrm{AA}} & M_{\mathrm{AB}} \\
M_{\mathrm{BA}} & M_{\mathrm{BB}}
\end{array}\right) \partial_{z}\left(\begin{array}{c}
\mu_{\mathrm{A}} \\
\mu_{\mathrm{B}}
\end{array}\right)\right]+\left(\begin{array}{c}
\sigma_{\mathrm{A}} \\
\sigma_{\mathrm{B}}
\end{array}\right)
$$

where $\mu_{m}$ is the exchange chemical potential of the solute $m$ and the solvent $S$ scaled to the thermal energy, and $M_{m n}=M_{n m}$ are symmetric mobility coefficients for the components $m, n=\mathrm{A}$, B. The mobility coefficients are dimensionless and scaled to the dimensionless length and time scales. This is also true for that accounts for solvent removal by evaporation at the surface. This means that $\sigma_{m}=0$ for all $0 \leq z<h$. We come back to this below.

We now briefly discuss the chemical potentials, mobilities, and source terms and refer for more extensive description to earlier work. ${ }^{7}$ The exchange chemical potentials are given by ${ }^{7}$

$$
\mu_{m}=\frac{\partial f}{\partial \phi_{m}}-\kappa_{m \mathrm{~A}} \partial_{z}^{2} \phi_{\mathrm{A}}-\kappa_{m \mathrm{~B}} \partial_{z}^{2} \phi_{\mathrm{B}}
$$

for the solutes $m, n=\mathrm{A}, \mathrm{B}$. Here, $f$ is a local free energy density, rendered dimensionless by dividing by the thermal energy and multiplying with our solvent volume scale. The gradient terms penalize inhomogeneities of the concentrations in the $z$ direction, where the $\kappa$ 's denote stiffnesses that in principle consist of contributions of energy and entropy. ${ }^{28}$ To highlight the role of dynamics, we do not explicitly include interactions of the solutes with the fixed substrate surface and the moving free surface. We return to the potential impact of surface interactions in our discussion below.

For the local free energy we use the standard Flory-Huggins theory for a ternary system, consisting of a monomeric solvent and two polymeric solutes of degrees of polymerization $N_{\mathrm{A}}$ and $N_{\mathrm{B}}$ for the two solutes A and B. We refer to refs 7, 29, and 30 for details. Interactions are described by the three FloryHuggins interaction parameters $\chi_{\mathrm{AB}}, \chi_{\mathrm{AS}}$, and $\chi_{\mathrm{BS}}$, the values of which we specify below.

Following de Gennes, we write for the stiffnesses $\kappa_{\mathrm{AB}}=\left(\chi_{\mathrm{AS}}\right.$ $\left.+\chi_{\mathrm{BS}}-\chi_{\mathrm{AB}}\right) / 6, \kappa_{\mathrm{AA}}=1 /\left(18 \phi_{\mathrm{A}}\right)+\chi_{\mathrm{AS}} / 3$, and $\kappa_{\mathrm{BB}}=\chi_{\mathrm{BS}} / 3{ }^{28}$ The concentration dependence of $\kappa_{\mathrm{AA}}$ accounts for entropy losses of our polymer A in a concentration gradient. ${ }^{28}$ For our component $\mathrm{B}$, in practice not actually a polymer but a fullerene derivative, we ignore this contribution.

The mobilities $M_{m n}$ in eq 1 depend on the tracer diffusivities and on the concentration of all three components, meaning they are local and in time varying quantities. We use the mobilities provided by the so-called slow-rather than by fast-mode theory, ${ }^{28,31}$ which we have discussed extensively in ref 7 and for that reason do not reproduce here. For the polymer tracer diffusivity, we assume free draining to apply because the polymer is relatively short, whereas for those of the smaller component and the solvent we use the usual StokesEinstein relation.

Finally, evaporation is in eq 1 described by the boundary condition 

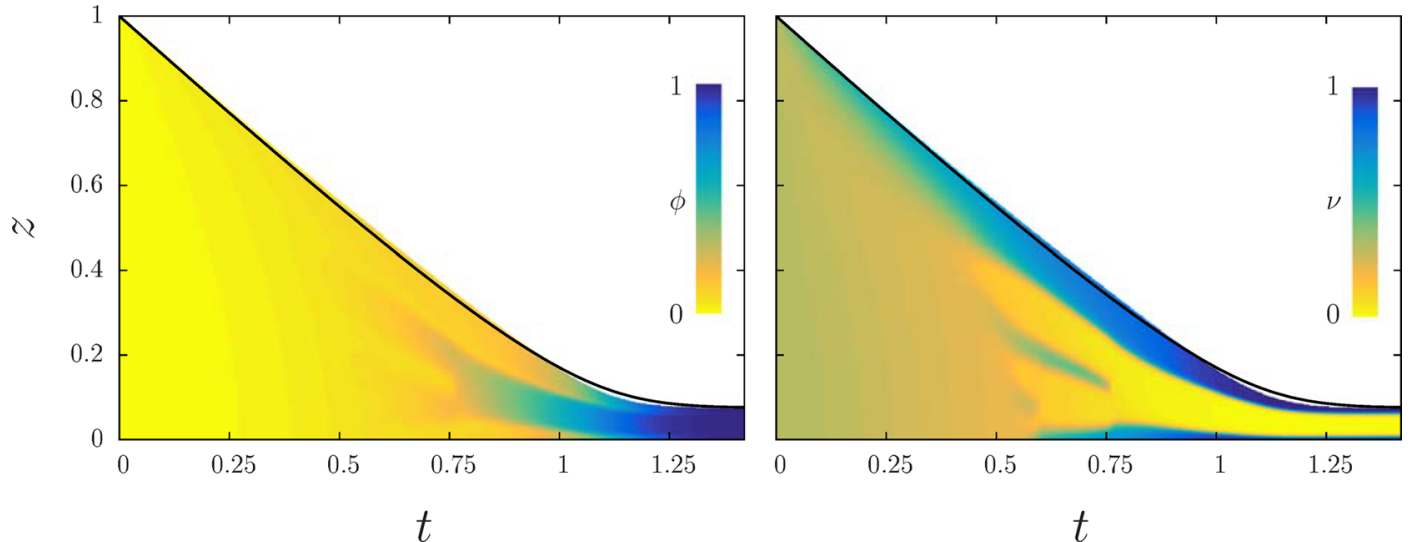

Figure 1. Predicted local blend concentration (left), $\nu$, and composition (right), $\phi$, in an evaporating model $\mathrm{DPP} / \mathrm{PCBM} / \mathrm{CHCl}$ solution as a function of the dimensionless vertical position $z$ and dimensionless time $t$ for a Biot number of 0.62 . See the main text. The composition is indicated by the color coding given on the right. The drawn line represents an approximate prediction for the layer thickness; see eq 4 and the main text.

$$
\sigma_{m}(h, t)=-\phi_{m}(h, t) j_{\text {evap }} \delta(z-h) / h_{0}
$$

(see refs $11,12,17$, and 23). The Dirac delta, $\delta$, ensures that mass accumulation due to evaporation takes place exclusively at the liquid-vapor interface. Consequently, at the surface the concentration, but not the composition, is affected by evaporation. For reasons of simplicity, we linearize the evaporation rate with respect to the surface concentration of solvent and obtain $j_{\text {evap }}=-\phi_{S}(h, t) .{ }^{11,12,17}$ We note that the rate of change of the surface concentration is coupled to the change in layer thickness by the equality $\mathrm{d}\left(h / h_{0}\right) / \mathrm{d} t=j_{\text {evap }}{ }^{12,32}$

\section{NUMERICAL RESULTS}

Now that we have presented all the ingredients of our model, we numerically solve eq 1 using standard finite-difference methods. ${ }^{23}$ To be able to do that, we have to assign values to all of our system parameters. The actual system that we have in mind in the context of solution casting of the active layer of an organic solar cell is the solvent chloroform, the polymer diketopyrrolopyrrole-quinquethiophene, and a $[6,6]$-phenyl $\mathrm{C}_{71}$-butyric acid methyl ester fullerene derivative. The former solute acts as an electron donor and the latter as an electron acceptor. Their effective degrees of polymerization and interaction parameters have been established experimentally in ref 5 , and we refer to that work for details. Hence, we take for the effective degrees of polymerization $N_{\mathrm{A}}=89, N_{\mathrm{B}}=7$, and $N_{\mathrm{S}}$ $=1$ and for the interaction parameters $\chi_{\mathrm{AB}}=1, \chi_{\mathrm{AS}}=0.1$, and $\chi_{\mathrm{BS}}=0.9$.

For our initial film thickness, $h_{0}$, we take 128 times the size of the chloroform molecule, i.e., approximately $65 \mathrm{~nm}$. For computational convenience we choose the thickness roughly 100 times smaller than in a typical experiment. This turns out not to be a drawback in our model system because the surface composition and structure are determined well before the film is dry. For the initial blend composition we set $\nu \equiv \phi_{\mathrm{A}} /\left(\phi_{\mathrm{A}}+\right.$ $\left.\phi_{\mathrm{B}}\right)=0.3$, which was found to be an optimal value for the production of organic solar cells. ${ }^{5,6}$ As the initial overall concentration we arbitrarily choose a value of $\phi \equiv \phi_{\mathrm{A}}+\phi_{\mathrm{B}}=$ 0.075 , which is well below the calculated spinodal concentration of 0.1202 and of comparable magnitude as typically used experimentally. ${ }^{6}$ In practice, initial concentrations are chosen such that the solution is stable and does not demix or gel.

As already announced, for the various tracer diffusivities we use estimates based on the relative sizes of the molecules in the solution. How these are connected to various cooperative diffusivities of the solutes as well as the solvent can be found in refs 7,12 , and 33, and we do not dwell on that here. The cooperative diffusivity of the solvent is important because it enters into the Biot number. This quantity is proportional to the ratio of the evaporation rate and this cooperative diffusivity, ${ }^{12}$ and its value indicates whether or not the solutes potentially accumulate at the free gas-liquid interface. If the Biot number is much smaller than unity, there is no evaporation-driven accumulation of solutes at that interface. In the present work, we consider Biot numbers slightly smaller than unity. This means that we focus on weak evaporationdriven concentration gradients. Our calculations show that this is sufficient to obtain selective enrichment of the free surface by one of the components.

Figure 1 shows our numerically obtained film thickness, local solute concentration, and the local blend composition within the film as a function of time. We find our layer thickness to be reasonably well described by the Bornside model (solid line), which predicts that the layer thickness obeys the following implicit relation $^{6,27}$

$$
h / h_{0}+\phi_{0} \ln \left[\left(h / h_{0}-\phi_{0}\right) /\left(1-\phi_{0}\right)\right]=-t
$$

with $\phi_{0}$ the initial uniform solute concentration. The Bornside model relies on the approximation that the film thickness decreases with a rate proportional to the overall concentration of the solutes rather than that in the top layer of the film.

In our case, evaporation-driven accumulation of solute near the interface in fact is absent, as is clear from the left panel of Figure 1. This explains the good agreement between the numerically calculated layer thickness and the prediction by eq 4. The slight disagreement for late times, that is, for times close to the drying time, is caused by accumulation of solvent, not solutes, near the interface. The solvent accumulation is driven by the phase separation of the two solutes. This can be inferred from the right panel of Figure 1, where we plot the local composition as a function of time.

We find that at early times the polymer displaces the fullerene near the free surface. The composition stratification that is a result of this is amplified at a later stage by entering the spinodal regime as a result of ongoing evaporation of solvent. This happens roughly at the dimensionless time $t \approx 0.4$. From then on a spinodal wave of alternating polymer-enrichment and -depletion layers propagates from the free surface toward the 
substrate. These layers subseqently coalesce, until the final morphology is formed at time $t \approx 0.7$. This structure consists of a fullerene-rich phase sandwiched in between two polymer-rich layers at the two interfaces. Our theoretical prediction for the final morphology resembles the experimentally observed one. ${ }^{5}$ Although our predicted enrichment at the substrate may vary with the initial layer thickness, the enrichment at the free surface is robust despite the fact that we did not include surface interactions. This means that the surface composition is not solely determined by thermodynamics but also by the dynamics.

\section{AN APPROXIMATE MODEL FOR PREDICTING SURFACE ENRICHMENT}

The fact that we are able to emulate what is seen experimentally does not imply that we have a clear view of the underlying physical principles on account of the complexity of the theory. A simple thought experiment allows us to pinpoint these and hence obtain an understanding of the physics at play. It seems reasonable to presume that the instantaneous evaporation of solvent at the surface only directly changes the surface concentration, but not the surface composition. The associated concentration gradient then gives rise to diffusion within the film. If the two components have different (cooperative) diffusivities, this in turn results in a composition gradient. Arguably, the gradients in composition are amplified at the point in time where the surface concentration reaches the spinodal region of the phase diagram.

Implementing this in our model description, we can simplify our kinetic equations (eq 1) by taking the following steps: (i) first we rewrite them in terms of the overall composition and concentration variables $\nu$ and $\phi$, (ii) we next linearize our generalized diffusion equation at the point in time where the spinodal concentration is reached, (iii) subsequently we suppress spatial gradients in the composition, and (iv) we insist that evaporation only directly affects the concentration gradient. As is detailed in the Supporting Information, this approximate treatment produces a single diffusion equation of the form $\partial_{t} \nu=\partial_{z} D_{\nu \phi} \partial_{z} \phi$. Here, $D_{\nu \phi}=D_{\nu \phi}\left(\nu, \phi_{\text {spin }}(\nu)\right)$ denotes a cooperative cross diffusivity that depends on the mean composition and the spinodal concentration, $\phi_{\text {spin, }}$ as well as the interaction parameters and the various tracer diffusivities (see Supporting Information). The simplified diffusion equation shows that a gradient in $\phi$ drives a gradient in $\nu$, not dissimilar to how in the Soret effect a gradient in temperature drives a gradient in concentration. ${ }^{34}$

The sign of $D_{\nu \phi}$ at the surface of the film, where the concentration of solutes is the highest, determines whether the gradient in composition follows the gradient in concentration (if $D_{\nu \phi}>0$ ) or is reversed by it (if $D_{\nu \phi}<0$ ). The sign of $D_{\nu \phi}$ is predominantly determined by the cooperative diffusivities $D_{\mathrm{AA}}$ and $D_{\mathrm{BB}}$ of the solutes $\mathrm{A}$ and $\mathrm{B}$ at the surface. If the fullerene (B) has the largest cooperative diffusivity $\left(D_{\mathrm{AA}}<D_{\mathrm{BB}}\right)$, the fullerene inhomogeneity moves faster from the free surface into the film than that of the polymer. As a result, $D_{\nu \phi}$ is positive and the surface concentration of the polymer increases relative to that of the fullerene.

We now inspect under what conditions $D_{\nu \phi}$ changes sign for our model mixture $\mathrm{DPP} / \mathrm{PCBM} / \mathrm{CHCl}_{3}$ and verify if under those conditions the fullerene rather than the polymer enriches the surface. We expect from Figure 2, where we present $D_{\nu \phi}$ as a function of the blend composition at the spinodal concentration as well as of the (off-)diagonal cooperative

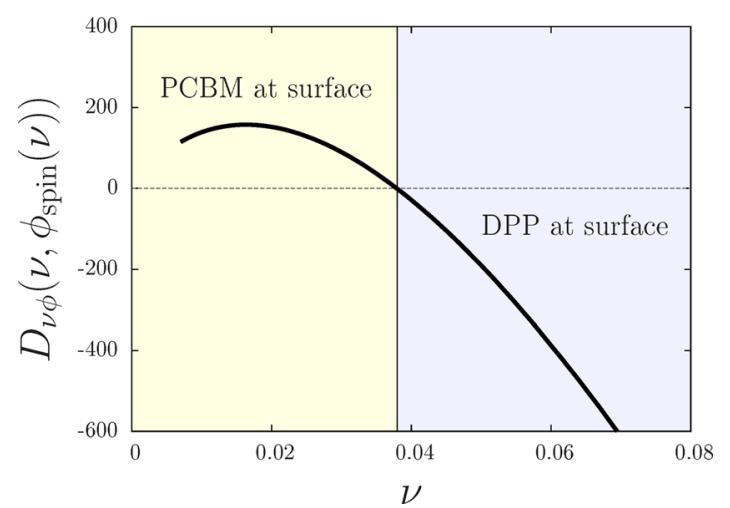

Figure 2. Dimensionless composition-concentration cross diffusivity $D_{\nu \phi}$ as a function of the overall composition, $\nu$, calculated at the spinodal concentration $\phi_{\text {spin }}(\nu)$ for the model mixture DPP/PCBM/ $\mathrm{CHCl}_{3}$. See main text and Supporting Information. If $D_{\nu \phi}$ is positive (for DPP fractions $\nu>0.038$ ), then the surface is expected to be enriched by DPP, whereas if it is negative (for $\nu<0.038$ ), then PCBM is expected to enrich the surface.

diffusivities, that this happens for polymer fractions lower than $3.8 \%$. We note that this fraction is $2.7 \%$ if fast-mode diffusion theory is used instead of slow-mode theory. We verify the slowmode prediction by solving the full diffusion equation in eq 1 for polymer fractions of $\nu=0.03$ and $\nu=0.05$ and an initial overall concentration of $\phi_{0}=0.11$. We present our findings in Figure 3, showing that our prediction is indeed correct. For the polymer fraction of 0.05 , we indeed find that DPP enriches the surface. However, for a polymer fraction of 0.03 PCBM enriches the free surface.

Notice that our approximate prediction for the layer thickness, eq 4, based on the assumption of uniformly dissolved material, and indicated in the figure by the solid line, reasonably accurately matches our numerical results. This is despite the obvious gradients in our numerical calculations. This means that agreement between the theoretical layer thickness in eq 4 and experimental data does not rule out vertical stratification.

\section{CONCLUSION}

In conclusion, we find that upon the evaporation of solvent from thin films containing two incompatible solutes surface enrichment by one of the two components need not to be solely due to a thermodynamic preference of that component for that interface. Indeed, a sufficiently large difference in the collective diffusivities of the two components is all that is needed to cause surface enrichment: the slowest component enriches the surface. The collective diffusivity of a component is on the one hand determined by its size, through the tracer diffusivity, and on the other hand by the thermodynamics of the mixture. This means that the smallest component need not necessarily be the fastest and that the solute composition may determine what component accumulates at the surface.

Our model calculations for the $\mathrm{DPP} / \mathrm{PCBM} / \mathrm{CHCl}_{3}$ solution, inspired by the experiments by Franeker et al., show that the polymer accumulates at the surface, unless the solute fraction of the polymer is sufficiently low (a few percent). We find this even though neither the polymer nor the fullerene has a preference for the surface in our model calculations. This means that experimentally encountered accumulation of the polymer at the free surface is not necessarily explained by thermodynamic arguments alone. In practice, preferential wetting of one of the components does of 

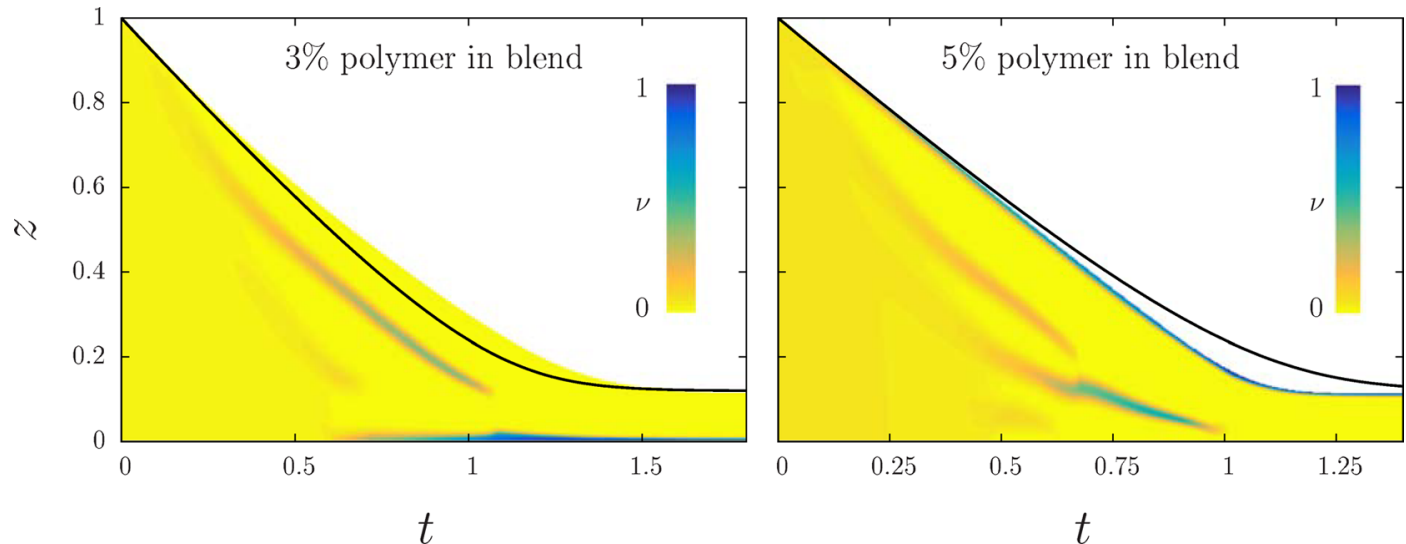

Figure 3. Predicted local blend composition, $\nu$, in an evaporating model DPP/PCBM/ $\mathrm{CHCl}_{3}$ solution as a function of the dimensionless vertical position $z$ and dimensionless time $t$. The overall blend compositions are 0.03 (left) and 0.05 (right). The composition is indicated by the color coding given on the right. The drawn line represents the layer thickness approximated by eq 4 (see the main text).

course also play a role. We have investigated the influence of preferential wetting on the critical polymer fraction below which the fullerene enriches the free surface. If the fullerene would preferentially wet the surface, this critical polymer fraction shifts to larger values, whereas this polymer fraction shifts to smaller values if the polymer preferentially wets the surface. The fraction at which this happens differs for slow- and fast-mode diffusion theory, indicating that the consequences of the commonly used incompressibility approximation should be studied in more detail, for instance using MD simulations.

In more general terms, our model calculations suggest some level of control over the surface composition of solvent-cast polymer blends, in particular via the solute composition. We expect that this level of control is largest for incompatible solutes with similar interactions with the liquid-gas interface and with similar molecular weights. Clearly, kinetic control over thin-film structuring merits further investigations, also experimental, given the importance of surface enrichment in the context of organic photovoltaics and thin-film organic electronics in general.

\section{ASSOCIATED CONTENT}

\section{S Supporting Information}

The Supporting Information is available free of charge on the ACS Publications website at DOI: 10.1021/acs.macromol.7b01224.

Steps used to obtain the diffusivities and the Biot number (PDF)

\section{AUTHOR INFORMATION}

\section{Corresponding Author}

*E-mail: charley.schaefer@durham.ac.uk (C.S.).

\section{ORCID}

J. J. Michels: 0000-0003-1591-4449

\section{Present Address}

C.S.: Department of Physics and Chemistry, Durham University, Durham DH1 3LE, UK.

\section{Notes}

The authors declare no competing financial interest.

\section{ACKNOWLEDGMENTS}

This research forms part of the research program of the Dutch Polymer Institute (DPI), project \#734.

\section{REFERENCES}

(1) Deibel, C.; Dyakonov, V. Polymer-fullerene bulk heterojunction solar cells. Rep. Prog. Phys. 2010, 73, 096401.

(2) Brabec, C. J.; Gowrisanker, S.; Halls, J. J. M.; Laird, D.; Jia, J.; Williams, S. P. Spin coating of a PMMA/chlorobenzene solution. Adv. Mater. 2010, 22, 3839-3856.

(3) Nelson, J. Polymer:fullerene bulk heterojunction solar cells. Mater. Today 2011, 14, 462-470.

(4) Greenham, N. C. Polymer solar cells. Philos. Trans. R. Soc., A 2013, 371, 20110414.

(5) Kouijzer, S.; Michels, J.; van den Berg, M.; Gevaerts, V. S.; Turbiez, M.; Wienk, M. M.; Janssen, R. A. J. Predicting morphologies of solution processed polymer:fullerene blends. J. Am. Chem. Soc. 2013, 135, 12057-12067.

(6) van Franeker, J. J.; Westhoff, D.; Turbiez, M.; Wienk, M. M.; Schmidt, V.; Janssen, R. A. J. Controlling the dominant length scale of liquid-liquid phase separation in spin-coated organic semiconductor films. Adv. Funct. Mater. 2015, 25, 855-863.

(7) Schaefer, C.; Michels, J. J.; van der Schoot, P. Structuring of thinfilm polymer mixtures upon solvent evaporation. Macromolecules 2016, 49, 6858-6870.

(8) Campoy-Quiles, M.; Ferenczi, T.; Agostinelli, T.; Etchegoin, P. G.; Kim, Y.; Anthopoulos, T. D.; Stavrinou, P. N.; Bradley, D. D. C.; Nelson, J. Morphology evolution via self-organization and lateral and vertical diffusion in polymer:fullerene solar cell blends. Nat. Mater. 2008, 7, 158-164.

(9) van Franeker, J. J.; Heintges, G. H. L.; Schaefer, C.; Portale, G.; Li, W.; Wienk, M. M.; van der Schoot, P.; Janssen, R. A. J. Polymer solar cells: Solubility controls fiber network formation. J. Am. Chem. Soc. 2015, 137, 11783-11794.

(10) Münch, A. M.; Please, C. P.; Wagner, B. Spin coating of an evaporating polymer solution. Phys. Fluids 2011, 23, 102101.

(11) Wodo, O.; Ganapathysubramanian, B. Modeling morphology evolution during solvent-based fabrication of organic solar cells. Comput. Mater. Sci. 2012, 55, 113-126.

(12) Wodo, O.; Ganapathysubramanian, B. How do evaporating thin films evolve? Unravelling phase-separation mechanisms during solvent-based fabrication of polymer blends. Appl. Phys. Lett. 2014, 105, 153104.

(13) Buxton, G. A.; Clarke, N. Ordering polymer blend morphologies via solvent evaporation. Europhys. Lett. 2007, 78, 56006.

(14) Jukes, P. C.; Heriot, S. Y.; Sharp, J. S.; Jones, R. A. L. Timeresolved light scattering studies of phase separation in thin film semiconducting polymer blends during spin-coating. Macromolecules 2005, 38, 2030-2032.

(15) Coveney, S.; Clarke, N. Breakup of a transient wetting layer in polymer blend thin films: Unification with 1D phase equilibria. Phys. Rev. Lett. 2013, 111, 125702. 
(16) Zoumpouli, G. A.; Yiantsios, S. G. Hydrodynamic effects on phase separation morphologies in evaporating thin films of polymer solutions. Phys. Fluids 2016, 28, 082108.

(17) Kim, C.-S.; Saylor, D. M.; McDermott, M. K.; Patwardhan, D. V.; Warren, J. A. Modeling solvent evaporation during the manufacture of controlled drug-release coatings and the impact on release kinetics. J. Biomed. Mater. Res., Part B 2009, 90B, 688-699.

(18) Morita, H.; Kawakatsu, T.; Doi, M. Dynamic density functional study on the structure of thin polymer blend films with a free surface. Macromolecules 2001, 34, 8777-8783.

(19) Coveney, S.; Clarke, N. Pattern formation in polymer blend thin films: Surface roughening couples to phase separation. Phys. Rev. Lett. 2014, 113, 218301.

(20) Björström, C. M.; Bernasik, A.; Rysz, J.; Budkowski, A.; Nilsson, S.; Svensson, M.; Andersson, M. R.; Magnusson, K. O.; Moons, E. Multilayer formation in spin-coated thin films of low-bandgap polyfluorene:PCBM blends. J. Phys.: Condens. Matter 2005, 17, L529-L534.

(21) Björström, C. M.; Nilsson, S.; Bernasik, A.; Budkowski, A.; Andersson, M.; Magnusson, K. O.; Moons, E. Vertical phase separation in spin-coated films of a low bandgap polyfluorene/ PCBM blend-effects of specific substrate interaction. Appl. Surf. Sci. 2007, 253, 3906-3912.

(22) Vaynzof, Y.; Kabra, D.; Zhao, L.; Chua, L. L.; Steiner, U.; Friend, R. H. Surface-directed spinodal decomposition in poly[3hexylthiophene] and $\mathrm{C}_{61}$-butyric acid methyl ester blends. ACS Nano 2011, 5, 329-336.

(23) Michels, J. J.; Moons, E. Simulation of surface-directed phase separation in a solution-processed polymer/PCBM Blend. Macromolecules 2013, 46, 8693-8701.

(24) Zhao, K.; et al. Vertical phase separation in small molecule:polymer blend organic thin film transistors can be dynamically controlled. Adv. Funct. Mater. 2016, 26, 1737-1746.

(25) Nikiforow, I.; Adams, J.; König, A. M.; Langhoff, A.; Pohl, K.; Turshatov, A.; Johannsmann, D. Self-stratification during film formation from latex blends driven by differences in collective diffusivity. Langmuir 2010, 26, 13162-13167.

(26) Zhou, J.; Jiang, Y.; Doi, M. Cross Interaction Drives Stratification in Drying Film of Binary Colloidal Mixtures. Phys. Rev. Lett. 2017, 118, 108002.

(27) Bornside, D. E.; Macosko, W. W.; Scriven, L. E. Spin coating of a PMMA/chlorobenzene solution. J. Electrochem. Soc. 1991, 138, 317320.

(28) de Gennes, P. G. Dynamics of fluctuations and spinodal decomposition in polymer blends. J. Chem. Phys. 1980, 72, 47565763.

(29) Flory, P. J. Principles of Polymer Physics; Cornell University Press: Ithaca, NY, 1981.

(30) Rubinstein, M.; Colby, R. H. Polymer Physics, 4th ed.; Oxford University Press: Oxford, 2003.

(31) Composto, R. J.; Kramer, E. J.; White, D. M. Fast macromolecules control mutual diffusion in polymer blends. Nature 1987, 328, 234-236.

(32) Ledesma-Aguilar, R; Vella, D.; Yeomans, J. M. LatticeBoltzmann simulations of droplet evaporation. Soft Matter 2014, 10, 8267-8275.

(33) He, D. Q.; Kwak, S.; Nauman, E. B. On phase equilibria, interfacial tension and phase growth in ternary polymer blends. Macromol. Theory Simul. 1996, 5, 801-827.

(34) Duhr, S.; Braun, D. Why molecules move along a temperature gradient. Proc. Natl. Acad. Sci. U. S. A. 2006, 103, 19678-19682. 